\title{
ESTUDIOS
}

\section{El itinerario laboral de los inmigrantes en España, de la irregularidad a la integración'}

\section{Manuel López-Casquete de Prado}

Resumen: La población inmigrante en situación irregular se caracteriza esencialmente por su alta vulnerabilidad como sujeto de derechos humanos y laborales. La regulación relativa a los derechos y libertades de los extranjeros en España efectuada por la L.O. 4/2000 contempla la incorporación al mercado de trabajo como el principal requisito necesario para la concesión de los permisos de residencia. Por este motivo consideramos de gran importancia conocer los obstáculos encontrados por la población inmigrante en su camino hacia la integración laboral, a fin de promover ésta y posibilitar para los inmigrantes un estatus jurídico que los iguale a la población autóctona como sujetos de derechos humanos. En este trabajo se hará revisión de algunos de los principales obstáculos para la integración laboral identificados por la doctrina, la cual será complementada con la opinión al respecto de algunos inmigrantes residentes en España.

Palabras clave: inmigración, irregular, integración, laboral, obstáculos.

Fecha de recepción: 6 de diciembre de $20 \mathrm{II}$.

Fecha de admisión definitiva: 10 de septiembre de 2012 .

\footnotetext{
' En el origen de este trabajo recibí interesantes sugerencias de los profesores $\mathrm{H}$. Molina y J. Ramírez
} que agradezco sinceramente. 
Obstacles for the integration of immigrants in the spanish labor market

\begin{abstract}
The illegal immigrant population is essentially characterized by its great vulnerability with respect to human and labor rights. The normative with respect to the rights and freedom of foreigners in Spain according to the LO 4/2000 makes incorporation into the labor market the main prerequisite for residency permits. For this reason we attach great importance to knowing the obstacles encountered by immigrants making their way towards integration into the labor market, to thus promote it and open up the possibility for immigrants to have a legal status equivalent to that of the native population as far as human rights are concerned. This paper reviews some of the main obstacles for labor integration identified in this document, and will supplement this with the views of some immigrants living in Spain.
\end{abstract}

Key words: immigration, irregular, integration, employment, obstacles.

\section{Obstacles à l'intégration des immi- grés dans le monde du travail en Espagne}

Résumé: La population immigrante en situation irrégulière se caractérise essentiellement par sa grande vulnérabilité en tant que sujet des droits de l'Homme et des droits du travail. La réglementation relative aux droits et libertés des étrangers en Espagne, soumise à la L.O. (Loi) 4/2000, considère l'intégration dans le marché du travail comme la principale condition pour obtenir le permis de séjour. Pour ce motif nous considérons essentiel de connaître les obstacles rencontrés par la population immigrante dans son cheminement vers l'intégration dans le monde du travail, afin de la promouvoir et de permettre aux immigrés d'avoir un statut juridique en égalité avec la population autochtone en tant que sujet des Droits de l'Homme. Nous reverrons dans ce texte certains des principaux obstacles identifiés par la doctrine à l'intégration dans le monde du travail, appuyés par l'opinion à ce sujet de quelques immigrés résidents en Espagne.

Mots Clés: immigration, irrégulière, intégration, emploi, obstacles.

\section{Introducción}

Uno de los debates sociales y políticos más vivos en la actualidad española gira en torno al fenómeno de la inmigración. La actual tasa de inmigrantes, superior al $12 \%{ }^{2}$, no sólo ha situado a España en cifras semejantes a las de los países tradi-

${ }^{2}$ Fuente: web del Instituto Nacional de Estadística (www.ine.es) consultada el 12 de abril de 2012. 
cionalmente receptores de inmigrantes, sino que se ha convertido, posiblemente, en la principal vía de transformación social y demográfica de nuestro país. ${ }^{3}$

Dentro del colectivo inmigrante, merece especial atención la situación de quienes se encuentran en régimen irregular por haber entrado en España de forma ilegal o por haber expirado sus visados o permisos de residencia temporales. Dicha población se caracteriza esencialmente por su alta vulnerabilidad como sujeto de derechos humanos y laborales ${ }^{4}$, situación que se agrava a causa de las redes de tráfico de personas. ${ }^{5}$

En esta línea, y con cierta ironía, Noll se refiere a la aporía de los derechos humanos:

los derechos humanos son prácticamente inaccesibles para los inmigrantes en situación irregular, pero seguimos reclamando que sean de aplicación universal. ¿Cómo es esto posible? ¿ ¿Será que la normativa produce esta inaccesibilidad por algún motivo técnico que me perdí en mis estudios? [... $]_{i} O$ será necesario investigar para derribar obstáculos legales y demostrar que los derechos humanos pueden ser reclamados de forma segura y exitosa también por los inmigrantes en situación irregular? ${ }^{6}$

Tjernberg, por su parte, describe a los inmigrantes irregulares como invisibles, mal pagados, desprotegidos y explotados. ${ }^{7}$ Ariza, Morales y Morales se refieren a este colectivo como hermética bolsa de irregulares en condiciones de marginalidad. ${ }^{8}$

La regulación relativa a los derechos y libertades de los extranjeros en España efectuada por la L.O. 4/2000 considera la incorporación al mercado de trabajo como el principal requisito para la concesión y mantenimiento de los permisos de residencia. De hecho, la exposición de motivos del reglamento que desarrolla la

${ }^{3}$ F. J. Moreno Y M. BRUquetas (2009).

4 J. BuSTAMANTE (2003).

${ }^{5}$ A. M. LóPEz (2003).

${ }^{6}$ G. Noll (2010:241 y 242).

${ }^{7}$ M. TJeRNBERG (2010:150).

8 J. A. Ariza, A. C. Morales y E. Morales (2010: 230). 
citada Ley Orgánica ${ }^{9}$ basa el estatus jurídico de los inmigrantes en la necesidad de cobertura de puestos de trabajo. Por este motivo es de gran importancia conocer los obstáculos encontrados por la población inmigrante en su camino hacia la integración laboral a fin de promover esta última y posibilitar para ellos un estatus jurídico que los iguale a la población autóctona como sujetos de derechos humanos.

Son numerosas las aportaciones doctrinales que insisten en la necesidad de trabajar por la integración laboral de los inmigrantes, en línea con el Informe sobre las Estrategias y los Medios para la Integración de los Inmigrantes en la Unión Europea. ${ }^{10}$ Dicho informe plantea que es necesario integrar a los inmigrantes que viven y trabajan entre nosotros, que no causan daño y que enriquecen nuestra vida. En la misma línea, otros autores afirman que sin integración no hay inmigración, sino explotación, discriminación y marginación. " Solanes, por su parte, sostiene que la devaluación de los derechos fundamentales de los inmigrantes no es más que una reducción en la calidad de nuestras democracias, una reformulación a la baja del Estado de Derecho que ha de ser cuestionada, criticada y combatida. ${ }^{12}$ También afirma que el extranjero en situación irregular se encuentra en el último peldaño de esta gradación de derechos. Dada la imperiosa necesidad de obtener un contrato de trabajo para regularizar su situación, el inmigrante se convierte en el blanco perfecto de la explotación empresarial, del arrendador de su vivienda o de cualquiera que con él se relacione. ${ }^{13}$

Así pues, dada la importancia del fenómeno migratorio en España, la especial vulnerabilidad de los inmigrantes en situación irregular y el papel clave del acceso a puestos de trabajo para la obtención de los permisos de residencia, consideramos oportuno plantear a través de este artículo una reflexión que ayude a conocer los principales motivos que dificultan el proceso hacia la integración laboral de los inmigrantes.

\footnotetext{
${ }^{9}$ RD 557/2011 de 20 de abril.

${ }^{10}$ Parlamento Europeo, Documento de Sesión, Final A6-0190/2006, 17 de mayo de 2006.

11 A. IZQUIERDO (2003)

${ }^{12}$ A. Solanes (2006: 6)

${ }^{13}$ A. Solanes $(2006: 13)$
} 
Creemos que constituye un auténtico imperativo ético profundizar en el drama de la exclusión social de los inmigrantes en situación irregular para tratar de ofrecer una respuesta-como sociedad de acogida- a los graves problemas que esto plantea. No debemos olvidar que, como afirmaba Martínez Martínez en esta misma revista, los verdaderos artífices de la integración no son los inmigrantes, sino las sociedades de acogida. ${ }^{14}$

La estructura de este texto responde al siguiente esquema: en el punto segundo se expone la metodología que seguiremos en este estudio; en el punto tercero se analizarán los obstáculos a la integración laboral identificados por la doctrina y se expondrá la visión al respecto de algunos inmigrantes entrevistados; finalmente, en el punto cuarto se expondrán las conclusiones de nuestro trabajo.

\section{Metodología}

Este estudio pretende sistematizar las conclusiones obtenidas por la literatura especializada en lo relativo al proceso de integración laboral de los inmigrantes. Para ello se ha hecho una revisión de la literatura con el propósito de obtener un listado de los obstáculos que, en opinión de la doctrina, influyen en dicho proceso. Una vez redactada esta parte del artículo, elaboramos dieciocho entrevistas en profundidad para conocer la visión al respecto de algunos inmigrantes inmersos en procesos de integración laboral. Dichas entrevistas, dadas las limitaciones de este estudio, no pretenden en modo alguno ofrecer evidencias empíricas sobre la validez de las propuestas de la doctrina. Muy al contrario, tan sólo se ofrecen como un intento de ampliar nuestro enfoque sobre las cuestiones objeto de estudio y de dar voz a quienes están personalmente implicados en este proceso.

Para la elaboración de las dieciocho entrevistas se tuvo en cuenta la existencia de tres grandes colectivos de inmigrantes extracomunitarios: subsaharianos, magrebíes e hispanoamericanos. Dentro de cada uno de estos grupos se entrevistó a seis personas (tres hombres y tres mujeres en cada caso), contemplándose ocho nacionalidades diferentes distribuidas según muestra la tabla $\mathrm{n}^{\circ} 1$.

14 J. Martínez Martínez (2006: 361) 
TABLA I

\begin{tabular}{|l|c|c|c|c|c|c|c|c|}
\cline { 2 - 9 } \multicolumn{1}{c|}{} & \multicolumn{4}{c|}{ HISPANOMMÉRICA } & \multicolumn{3}{c|}{ MAGREB } & ÁFRICA SUBSAHARIANA \\
\cline { 2 - 9 } \multicolumn{1}{c|}{} & Perú & Ecuador & Bolivia & Paraguay & Marruecos & Argelia & Senegal & Camerún \\
\hline HOMBRES & & 1 & & 2 & 3 & & 3 & \\
\hline MUJERES & 1 & 1 & 1 & & 2 & 1 & 2 & 1 \\
\hline
\end{tabular}

Elaboración propia

A fin de obtener opiniones de personas en distinta situación dentro del proceso de integración, se ha entrevistado a inmigrantes en muy diversas condiciones personales, jurídicas y laborales: desde el punto de vista jurídico, se ha entrevistado tanto a personas en situación regular como en situación irregular; en lo relativo a la situación laboral, hemos entrevistado a desempleados, empleados en el sector informal (básicamente en la venta ambulante), empleados sin contrato, empleados con contrato en vigor y titulares de negocios propios. También desde el punto de vista de la formación se ha entrevistado a personas en distintas situaciones: inmigrantes que han cursado Educación Primaria, Educación Secundaria, Formación Profesional $u$ otros títulos, Bachillerato y estudios Universitarios. El rango de edad de los entrevistados oscila entre los 27 y los 47 años. Las entrevistas se realizaron en la ciudad de Sevilla en julio de 2011 con el apoyo de la Asociación "Casa de Todos" y de la ONG Claver, miembro del Servicio Jesuita a Migrantes.

\section{Obstáculos en el proceso de integración laboral}

Los obstáculos planteados por la literatura se centran fundamentalmente en cinco aspectos: discriminación (por ser inmigrante o por motivos de raza, sexo, procedencia o religión), papel de las AA.PP. (deficiencias en su funcionamiento, complejidad normativa, inexistencia de recursos y orientación para el autoempleo), falta de formación (estudios, dominio del idioma y homologaciones), condiciones económicas y empresariales adversas (paro estructural, economía sumergida y prácticas abusivas) y círculo vicioso de la inmigración irregular. En la tabla 2 se ofrece un esquema de las aportaciones doctrinales halladas respecto a cada una de estas cuestiones. 


\section{TABLA 2. Obstáculos a la integración laboral: revisión de la literatura}

\begin{tabular}{|c|c|}
\hline OBSTÁCULOS & AUTORES \\
\hline $\begin{array}{l}\text { Discriminación por el hecho de ser inmigrante } \\
\text { o por razón de procedencia, raza, sexo y } \\
\text { religión }\end{array}$ & 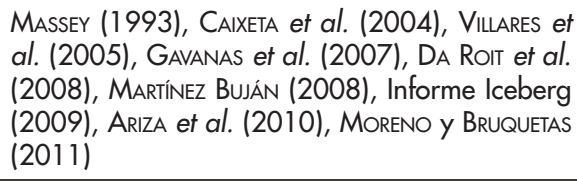 \\
\hline $\begin{array}{l}\text { Papel de las AA.PP. (deficiencias en su funciona- } \\
\text { miento, complejidad normativa, inexistencia de } \\
\text { recursos y orientación para el autoempleo) }\end{array}$ & $\begin{array}{l}\text { LALONDE y TOPEL (1997), MASSEY y DURAND (2003), } \\
\text { LÓPEZ (2005), Informe Iceberg (2009) }\end{array}$ \\
\hline $\begin{array}{l}\text { Falta de formación, deficiente manejo del idioma } \\
\text { y dificultad para homologación de titulaciones }\end{array}$ & $\begin{array}{l}\text { ANEAS y DONOSO (2008), VILLAGÓmEZ y OTERO } \\
\text { (2008), Informe lceberg (2009) }\end{array}$ \\
\hline $\begin{array}{l}\text { Condiciones económicas y empresariales } \\
\text { adversas (existencia de economía sumergida, } \\
\text { desempleo estructural en España, prácticas } \\
\text { abusivas por parte de los empresarios) }\end{array}$ & $\begin{array}{l}\text { GOZALVES (1995), AlAÓN Y GÓmEz (2004), BALDWIN- } \\
\text { EDWARDSY ARANGO (2009), Informe lceberg (2009), } \\
\text { SCHNEIDER (2010), Círculo de Empresarios (2010), } \\
\text { MORENO Y BRUQUETAS (201 1) }\end{array}$ \\
\hline Círculo vicioso de la inmigración & $\begin{array}{l}\text { Solé et al. (2000), BONILLA (2003), BazZaCO } \\
(2008)\end{array}$ \\
\hline
\end{tabular}

Elaboración propia.

Analizamos a continuación de forma más detallada cada uno de estos obstáculos.

3. I. Discriminación por el hecho de ser emigrante o por razón de raza, sexo, procedencia o religión

Aunque se trata de motivos de discriminación conceptualmente distintos, a menudo es imposible disociarlos, ya que se funden en una única imagen social. Por este modo los planteamos como un único epígrafe, aunque en nuestras entrevistas se plantearon separadamente.

Ariza, Morales y Morales ${ }^{15}$ han detectado actitudes significativas de discriminación laboral por razón de procedencia entre los inmigrantes extracomunitarios

15 J. A. Ariza, A. C. Morales y E. Morales, (2010: 222). 
(el 16,8\% de su muestra). En el Informe Iceberg ${ }^{16}$, elaborado por la plataforma Andalucía Acoge, el porcentaje de inmigrantes que afirma sentirse discriminado por razón de sexo, etnia, procedencia, religión u otro motivo llega al $41 \%$. Las discriminaciones expresadas por las mujeres se centran casi exclusivamente en motivos de género y por el hecho de ser inmigrantes. En cuanto a los hombres, mencionan discriminación ideológica, por el hecho de ser inmigrantes y por razones de procedencia, raza y religión.

El alto volumen de discriminación por razón de sexo manifestado por las mujeres merece explicación detallada. Aunque se trata de una vía de discriminación que, al menos en principio, podría afectar en la misma medida a mujeres extranjeras y a autóctonas, la realidad ofrece perfiles más complejos.

Según Moreno y Bruquetas ${ }^{17}$, el mercado de trabajo español lleva a cabo un proceso de segmentación laboral en función de sexo, nacionalidad y ámbito de actividad, lo cual explica el diferente impacto del desempleo sobre los distintos colectivos de inmigrantes.

Así mismo, y como expresa la Teoría de las Causas Acumulativas ${ }^{18}$, el fenómeno migratorio ocasiona alteraciones y cambios en las sociedades emisoras y receptoras. Cuando los inmigrantes ocupan puestos bajos en la cadena laboral, los trabajadores nacionales adquieren el hábito de no ocupar dichos trabajos, incluso en épocas de elevado paro. Esto hace que sea necesario ocuparlos con mano de obra inmigrante.

Ambos efectos, segmentación del mercado por razón de sexo, nacionalidad yámbito de actividad, y renuencia de las personas autóctonas a ocupar puestos de trabajo menos valorados desempeñados en los últimos años por inmigrantes, se cumplen ampliamente en lo relativo al sector de limpieza de hogar y cuidado de personas dependientes, ocupado en un altísimo porcentaje por mujeres inmigrantes. Esta especialización también ha supuesto que el colectivo empleado en dichos sectores haya sufrido en menor medida los efectos de la recesión. ${ }^{19}$

\footnotetext{
${ }^{16}$ Informe lceberg (2009: 180).

17 Moreno, F. J. y BRuquetas, M. (2011).

${ }^{18}$ MASSEY, D. S. (1993).

${ }^{19}$ Moreno, F. J. y Bruquetas, M. (2011).
} 
Según Moreno y Bruquetas ${ }^{20}$, a 31 de diciembre de 2008, el 65,3\% de las inmigrantes empleadas en el sector eran de procedencia latinoamericana, predominando las nacionalidades ecuatoriana, boliviana y colombiana. Entre las extranjeras, las recién llegadas y las que están en situación irregular se ocupan de las modalidades de empleo peor valoradas socialmente.

En resumen, podemos afirmar que las inmigrantes se introducen en el país de acogida a través de trabajos más precarios, habitualmente como internas, para buscar en momentos posteriores trabajos en régimen externo. A medida que pueden ahorrar y regularizar su situación, pueden también reagrupar a su familia.

El colectivo de cuidadoras internas es, a buen seguro, uno de los más preocupantes en lo relativo al recorte de derechos que sufren los inmigrantes en situación irregular: horarios de trabajo extenuantes, escasa vida privada, desprotección social, imposibilidad de regularización administrativa... A todo ello hay que añadir un nivel salarial indigno y abusivo que, según datos obtenidos por Martínez Buján ${ }^{21}$ en Galicia y Navarra, oscila entre los 400 y los 1000 euros mensuales.

En cuanto a las entrevistas elaboradas por nosotros, existe un amplio consenso entre los entrevistados en afirmar que se sienten discriminados por el hecho de ser inmigrantes. En general, consideran que en los procesos de selección laboral se prefiere a los nacionales antes que a los extranjeros:

Existe mucha discriminación hacia los inmigrantes. La gente tiene una imagen falsa de la inmigración, y esto nos ocasiona muchos problemas. Creo que los empleadores prefieren a españoles cuando se va a dar de alta al trabajador y las condiciones de trabajo son buenas. Si las condiciones no son buenas o no les darán de alta, se prefiere a inmigrantes. O sea, se nos prefiere para trabajos precarios.

(Florence, mujer camerunesa de 44 años con formación universitaria).

En España se prefiere dar un trabajo a un paisano mejor que a un inmigrante. Hice un curso privado con prácticas en empresas y concedieron prácticas a los tres españoles que hicieron el curso y no las hubo para mí. Para el empresario es más ventajoso contratar a un español.

(Hasna, mujer marroquí de 27 años con título de Formación Profesional).

${ }^{20}$ Moreno, F. J. Y Bruquetas, M. (2011).

${ }^{21}$ Martínez Buján (2008), citado por Moreno, F. J. y Bruquetas, M. (2011). 
Los entrevistados también fueron preguntados sobre si existe discriminación por razón de raza, procedencia y religión. Las opiniones a este respecto están divididas, pero se observa con claridad que las personas procedentes del Magreb o países Subsaharianos experimentan más ese rechazo. ${ }^{22}$

Hay inmigrantes que se ven obligados a mentir diciendo que no son de un país musulmán para obtener un trabajo.

(Nana, mujer marroquí de 39 años con título de enseñanza secundaria).

Me pidieron que fuera a hacer una entrevista pero cuando vieron que era de raza negra se negaron a entrevistarme.

(Ndeye, mujer senegalesa de 39 años con título de enseñanza secundaria).

Creo que los inmigrantes con más dificultades son los africanos, ya que los empleadores prefieren a los latinos.

(Florence, mujer camerunesa de 44 años con formación universitaria).

La gente no se cree que soy marroquí, y por eso el trato es bueno; si yo llevara pañuelo y otro modo de vestir tendría más problemas.

(Hasna, mujer marroquí de 27 años con título de Formación Profesional).

Otro de los posibles motivos de discriminación sobre el que se interrogó a los inmigrantes fue el relativo al sexo. Casi todos los entrevistados contestaron que no existe tal discriminación, pero sí hacen hincapié en cómo los distintos sectores de actividad están preferentemente reservados a hombres (construcción, fontanería, electricidad...) o a mujeres (cuidados del hogar). Muchos de ellos manifestaron que, en la actual coyuntura, es más fácil encontrar empleo para las mujeres:

Es más difíiil trabajar para los hombres, ya que las mujeres podemos encontrar trabajo como empleadas de hogar.

(Gloria, mujer boliviana de 27 años con formación universitaria).

22 Esta idea es coherente con los estudios de CAIXETA et al. (2004) y GavanAs et al. (2007), citados por MORENO, F. J. Y BRUQUetAS, M. (2011), que han detectado actitudes muy significativas de rechazo hacia los magrebíes y subsaharianos. 


\subsection{Papel de las Administraciones Públicas}

3.2.I.Deficiencias en el funcionamiento de las administraciones públicas (AA.PP.) (Extranjería, Ayuntamientos, Servicios Sociales, Servicio Público de Empleo Estatal (SEPE) y organismos autonómicos de promoción del empleo).

Según expresa la Exposición de Motivos de la última modificación de la L.O. 4/2000 de 11 de enero sobre Derechos y Libertades de los Extranjeros en Espa$\tilde{n} a^{23}$, la normativa vigente se basa en los principios de igualdad en los derechos y obligaciones, integración y respeto a las normas básicas de la convivencia.

Las AA.PP., por tanto, deben ser instrumentos al servicio de estos principios; así, la Secretaría General de Inmigración y Emigración debe facilitar los medios legales que propicien una paulatina integración de los inmigrantes. Del mismo modo, los Ayuntamientos, a través del padrón y los distintos servicios sociales, deben contribuir a este objetivo general de la Ley en sus respectivas jurisdicciones. Por último, los servicios de promoción del empleo (SEPE y los organismos autonómicos competentes) están llamados a ser dinamizadores del mercado de trabajo y a mejorar las posibilidades de empleabilidad de toda la población.

Sin embargo, la doctrina revisada ofrece con frecuencia un planteamiento crítico respecto al papel de las AA.PP. en el proceso de integración laboral de los inmigrantes.

El informe Iceberg ${ }^{24}$ expone que un alto porcentaje de los casos estudiados manifiesta no contar con mecanismos formales de búsqueda de empleo, sino que principalmente recurren a la propia red informal de contactos o familiar. El recurso al SEPE se lleva a cabo exclusivamente para solicitar prestaciones o inscribirse como demandante de empleo, sin que se haya recogido ningún caso en el que realicen la inscripción como demandantes de mejora de empleo.

Existe una parte reducida de la muestra que ha obtenido empleo en una ocasión al menos a través de los servicios públicos de empleo, principalmente en Madrid y Cataluña. Los inmigrantes consultados manifiestan que la búsqueda de empleo es más eficaz a través de contactos, amistades y redes sociales, o bolsas de empleo

${ }^{23}$ RD 557/2011 de 20 de abril.

${ }^{24}$ Informe lceberg (2009) 
de entidades sociales y ONG. Muchos de ellos consideran que acudir a las oficinas públicas de empleo es una pérdida de tiempo.

En el caso de las entrevistas elaboradas por nosotros, la valoración sobre las distintas instituciones de la Administración Pública es muy diversa, y no es posible hallar un patrón claro de respuesta. Preguntados por la utilidad del Servicio Andaluz de Empleo (SAE) de cara a conseguir un empleo, tan sólo una persona manifestó haber encontrado trabajo por esta vía. El resto de los entrevistados, pese a haber elaborado sus curriculums y haber realizado entrevistas en el SAE, nunca han sido llamados.

Algunos entrevistados también expresaron quejas sobre el funcionamiento de la Administración:

En extranjería o en el SEPE te informan de distinta manera según a quién le preguntes. Además, cualquier trámite vale mucho dinero y son tasas muy caras para el poder adquisitivo de los inmigrantes. Lo mejor de la Administración es la sanidad.

(Hasna, mujer marroquí de 27 años con título de Formación Profesional).

Los inmigrantes sufren mucho y se les exige mucho. Los trámites para obtener y renovar su documentación son muy complicados.

(Ana Isabel, mujer peruana de 46 años con título de Formación Profesional).

\subsubsection{Complejidad normativa}

La principal diferencia para la integración laboral de los extranjeros en comparación con el itinerario de las personas autóctonas se basa en la normativa de extranjería, según el Informe Iceberg ${ }^{25}$. En la misma línea, Lalonde y Tope ${ }^{26}$ afirman que, en principio, la normativa sobre migraciones debería determinar los rumbos migratorios con más fuerza que los condicionantes económicos.

De ese modo, las políticas migratorias pueden ser calificadas como políticas de acceso selectivo que determinan la elegibilidad de los candidatos para la inmigración y condenan a ciertas categorías de inmigrantes a la clandestinidad ${ }^{27}$.

25 Informe lceberg (2009: 233).

${ }^{26}$ LALONDE Y TOPEL (1997).

27 MASSEY y DuRAND (2003), citado por LóPEZ, A.M. (2005) 
La complejidad normativa, que perjudica la seguridad jurídica, y el contenido restrictivo de la misma deben ser necesariamente tenidos en cuenta en nuestro estudio de cara a identificar barreras a la integración laboral. Interrogados sobre este particular, la gran mayoría de los entrevistados manifestaron que percibían como un problema dicha complejidad:

La legislación es muy complicada y cambiante, lo que nos genera mucha ansiedad porque cambian los requisitos para renovar los permisos de residencia y trabajo.

(Luis, hombre ecuatoriano de 45 años con título de Formación Profesional).

Las leyes nos parecen confusas, ya que cambian con mucha frecuencia, en ocasiones varias veces en el mismo año. Esto nos hace muy difícil conocerlas.

(Florence, mujer camerunesa de 44 años con formación universitaria).

\subsubsection{Inexistencia de recursos u orientación para el autoempleo}

El Informe lceberg ${ }^{28}$ plantea que la opción por el autoempleo es a veces la estrategia elegida por los inmigrantes para asegurar los meses de cotización a la Seguridad Social requeridos por la normativa para obtener las renovaciones de los permisos de residencia y trabajo sin depender de ningún empleador y, además, obtener una situación laboral digna y una fuente de ingresos suficiente para el mantenimiento de la unidad familiar.

Preguntados a este respecto en nuestras entrevistas, los inmigrantes responden mayoritariamente que no disponen de capital necesario ni conocen servicios de orientación o ayuda. Algunos también hacen referencia a la presión fiscal que tienen que soportar las empresas, que a menudo conocen por la experiencia de familiares o amigos.

La Administración cobra tasas muy altas por todo. Además, las empresas tienen que soportar muchos impuestos.

(Hassan, hombre marroquí de 45 años con formación universitaria).

Para montar un negocio hay que estudiarlo bien; tenemos la experiencia de algunos amigos que tienen negocios. Estas personas nos dicen que tienen que pagar muchos impuestos.

(Florence, mujer camerunesa de 44 años con formación universitaria).

${ }^{28}$ Informe lceberg (2009: 112). 


\subsection{Falta de formación, deficiente manejo del idioma y dificultad para homo- logar titulaciones}

Numerosos estudios han destacado la importancia de la formación en el proceso de integración laboral. ${ }^{29}$ En contra de los estereotipos habituales, la muestra de población extranjera analizada por el Informe Iceberg $^{30}$ ofrece datos que muestran una elevada formación de los inmigrantes: el 97,62\% cuenta con formación reglada, y el $38,10 \%$ ha cursado estudios universitarios.

Una vez alcanzado territorio español, apenas les resulta posible cursar formación ni ocupacional ni continua, ya que dedican casi todo su tiempo a buscar trabajo o a trabajar. ${ }^{31}$

Como consecuencia de los datos anteriormente expuestos, cabe señalar que el estereotipo del inmigrante como una persona con escaso nivel de formación y baja cualificación no es acertado. En cambio, debemos suponer que los países emisores envían como emigrantes a los miembros más capacitados de sus sociedades, los únicos que pueden asumir el riesgo del proceso con ciertas garantías de éxito. No olvidemos que dicho éxito repercutirá de forma clara en las familias de origen a través de la recepción de remesas.

Casi todos los inmigrantes entrevistados por nosotros coinciden en destacar la gran importancia de la formación de cara a acceder al mercado de trabajo. Incluso los universitarios consideran que tienen que formarse permanentemente para avanzar en su camino laboral.

Creo que si hubiera tenido un título universitario me habrían contratado en un proceso de selección en el que quedaron varios puestos vacantes.

(Hasna, mujer marroquí de 27 años con título de Formación Profesional).

El dominio del idioma merece consideración aparte. Es evidente que un manejo deficiente del idioma español merma las posibilidades de integración de los inmigrantes, no sólo para acceder al mercado de trabajo, sino también por el hecho de que la principal vía de regularización de los inmigrantes reconocida por la L.O.

29 Villagómez, E. y Otero, E. (2008), Aneas, A. y Donoso, T. (2008).

30 Informe lceberg (2009: 120).

${ }^{31}$ Informe lceberg (2009: 121). 
$4 / 2000$ y sus reglamentos de desarrollo, el arraigo social y laboral, exige de los inmigrantes un correcto dominio del idioma. Es, por tanto, de gran importancia conocer la situación a este respecto.

Algunos de nuestros entrevistados destacaron la importancia del idioma, la falta de dominio de este aspecto y la voluntad de mejorarlo.

Es cierto que muchos inmigrantes no dominan el idioma, pero les veo mucha voluntad de aprender. Los inmigrantes que llevan más tiempo enseñan a los demás; entre 6 y 9 meses después de llegar, ya se manejan con mucha soltura; la necesidad nos obliga.

(Florence, mujer camerunesa de 44 años con formación universitaria).

Un último aspecto debe ser mencionado en lo relativo a la formación: la posibilidad de homologar titulaciones cursadas en origen. Según Aneas y Donoso, las diferencias en cuanto a las estrategias de integración y sus logros están más determinadas por los niveles de cualificación en el país de origen que por las propias diferencias culturales..$^{32}$ A pesar de la importancia de la formación cursada en origen, a muchos inmigrantes no se les reconoce administrativamente ni es valorada por parte de las empresas a las que pretenden acceder. Muy por el contrario, las empresas suelen desconfiar acerca de la formación y la vida laboral desarrollada en el país emisor, incluso si puede acreditarse con certificados expedidos en origen. Más concretamente, de la muestra analizada por el Informe lceberg, casi el $40 \%$ posee estudios universitarios en origen. De este grupo se encuentran en proceso de homologación un $14 \%$ y la han obtenido sólo un $6 \%$.

La valoración ofrecida por los inmigrantes respecto al proceso de homologación incide en la dificultad burocrática del procedimiento, donde frecuentemente deben hacer frente al pago de elevadas tasas tanto en recepción como en origen. También se destaca el excesivo tiempo que demoran los trámites y la incertidumbre sobre si el proceso de homologación será o no exitoso.

En cuanto a nuestras entrevistas, tan sólo tres personas declaran haber conseguido una homologación parcial de sus estudios, manifestando que fue un proceso difícil y caro.

32 Aneas, A. y Donoso. T. (2008). 


\subsection{Condiciones económicas y empresariales adversas}

\subsection{Desempleo estructural en España}

Según la EPA, España ofrece unas tasas de desempleo cercanas al $23 \%$ y un número de desempleados próximo a los cinco millones trescientos mil. ${ }^{33} \mathrm{Es}$ evidente que semejante situación de crisis laboral debe afectar a los inmigrantes con especial intensidad. Como afirman Moreno y Bruquetas ${ }^{34}$, si los inmigrantes cubrieron gran parte del empleo creado en la época de crecimiento, también han asumido una parte desproporcionadamente alta del desempleo generado por la crisis, actuando como "colchón amortiguador" que ha protegido en parte a la población autóctona, ya que la tasa de paro soportada por el colectivo inmigrante es notablemente superior a la soportada por la población autóctona.

La gran mayoría de nuestros entrevistados hizo referencia a la situación de crisis y a las altas cifras de paro que soporta la economía española. Esta circunstancia es vista como uno de los mayores problemas de cara a que ellos puedan recibir oportunidades laborales. Una de las más graves consecuencias de esta situación es la precarización de los puestos de trabajo:

Creo que el gran problema para nosotros es el paro. Antes, hace diez años, había más trabajo y menos gente en el paro, y menos inmigrantes. Ahora todo es mucho más difícil. Por ejemplo, para entrar a trabajar en el hogar, una señora hace 6 ó 7 entrevistas. Todo esto ha hecho empeorar las condiciones de trabajo. Yo no trabajaría 12 horas por menos de 600 euros, pero algunos inmigrantes sí lo aceptan; entonces si yo me quedo sin trabajo me vería obligada a aceptar eso. El paro ha rebajado el valor del trabajo.

(Hasna, mujer marroquí de 27 años con título de Formación Profesional).

Dicha precarización de las condiciones de trabajo es especialmente dramática entre las empleadas de hogar internas:

Existen muchos abusos contra los que no tienen todavía papeles. Además, las mujeres que trabajan internas como empleadas de hogar no tienen derecho a nada. Comienzan a trabajar las primeras y son las últimas en acostarse.

(Nana, mujer marroquí de 39 años con título de enseñanza secundaria).

33 Datos procedentes de la página oficial de la EPA, www.ine.es, consultada el 30 de marzo de 2012.

${ }^{34}$ Moreno, F.J. y Bruquetas, M. (2011). 
En el empleo de hogar nos hacen de todo porque saben que tenemos miedo. Por eso los empleadores se permiten muchos abusos: en el horario, en el trato, en el sueldo...

(Florence, mujer camerunesa de 44 años con formación universitaria).

\subsubsection{Existencia de economía sumergida}

El fenómeno de la inmigración cobra perfiles propios en regiones fronterizas como Andalucía, especialmente si disponen de una economía sumergida significativa. ${ }^{35}$ Conviene recordar que los países del Sur de la Unión Europea tienen una mayor proporción de economía informal e inmigración irregular, y se ha establecido un nexo causa-efecto bilateral entre ambos fenómenos. ${ }^{36}$

Una parte importante de las oportunidades de empleo para los trabajadores inmigrantes se halla, por tanto, en la economía sumergida ${ }^{37}$. Según Moreno y Bruquetas $^{38}$, se estima que en España dicha economía sumergida oscila entre un 20 y un $23 \%$ de nuestro PIB. Este dato sitúa a España, junto con Grecia e Italia, entre los países de mayor economía sumergida de la OCDE, organización cuya media se situaba aproximadamente en el $14 \%$ en $2010 .{ }^{39}$

Los sectores de actividad que concentran mayores niveles de economía sumergida son fundamentalmente agricultura, construcción, algunos sectores de la industria manufacturera (textil, calzado y juguetes) y servicios (restauración, limpieza, servicio doméstico y tareas de cuidado). ${ }^{40}$

El acceso a empleos en la economía sumergida se convierte con frecuencia para los inmigrantes en la única alternativa. Esta situación incide muy negativamente en sus condiciones laborales, haciéndolas más precarias y abocándolos a una situación de desprotección derivada del hecho de no cotizar a la Seguridad

${ }^{35}$ Godenau, D. (2003)

${ }^{36}$ BALDWIN-EDWARDS, M. (2002)

${ }^{37}$ Círculo de Empresarios (2010), citado por MOREno, F.J. y BruQuetas, M. (2011).

${ }^{38}$ Alaón y Gómez (2004), citado por Moreno, F.J. y Bruquetas, M. (2011).

39 Schneider (2010), citado por Moreno, F.J. y Bruquetas, M. (2011).

40 Baldwin-Edwards y Arango (2009), citado por Moreno, F.J. y Bruquetas, M. (2011). 
Social, lo cual los hace más vulnerables ante los riesgos del ciclo vital asociados al empleo. ${ }^{41}$

Además, en el caso de los inmigrantes en situación irregular, les impide obtener un contrato en vigor con el que regularizar su situación, por lo que permanecen en el último escalón de la sociedad en su consideración como sujetos de derechos humanos.

Los datos de la EPA muestran que la tasa de regularidad en los empleos domésticos oscila entre el 20 y el $45 \%$, en función de las coyunturas relativas a la política migratoria, y que la parte más importante de dicho empleo permanece en la economía sumergida (tanto en el caso de empleadas domésticas autóctonas como inmigrantes).

Gran parte de nuestros entrevistados considera que la existencia de economía sumergida supone para ellos un grave problema, ya que no sólo incide en la precarización de los contratos, sino que dificulta las posibilidades de acceder a los permisos de residencia y trabajo.

\subsubsection{Prácticas abusivas por parte de los empresarios}

En julio de 2011, la prensa recogía la detención de 18 personas en Almería imputadas por organizar una red que vendía a los inmigrantes contratos falsos de trabajo utilizando los datos de 32 empresas sin el consentimiento de sus administradores. ${ }^{42}$ En este caso, los estafados eran no sólo los inmigrantes, sino también las empresas cuyos datos eran utilizados por esta red. Sea como sea, la compraventa de contratos es una práctica fraudulenta que aprovecha la urgencia de los inmigrantes por obtener un contrato de trabajo que les permita regularizar su situación a cambio de cantidades exorbitantes.

El Informe lceberg recoge otras formas de abuso por parte de las empresas. En el ámbito del empleo doméstico, por ejemplo, es frecuente que, pese a existir contrato en vigor y alta en la Seguridad Social, la parte de la cuota correspondiente al empleador sea efectivamente abonada por el empleado. ${ }^{43}$

${ }^{41}$ Moreno, F.J. y Bruquetas, M. (2011).

${ }^{42}$ Diario ABC Almería (2/07/2011).

${ }^{43}$ Informe lceberg (2009: 113-114). 
Además, dicho Informe lceberg recoge hasta seis modalidades de pago por conseguir trabajo ${ }^{44}$ : agencias de colocación informales, ETTs, aceptación de salarios más bajos, contratos de formación, pago de la SS correspondiente al empresario por el trabajador y compra de trabajo a nivel ideológico y emocional. A esto habría que añadir la compra mediante contraprestación monetaria, como con frecuencia sucede a los inmigrantes, como vimos en el caso de la noticia recogida por el diario $A B C$.

En el caso de los inmigrantes entrevistados por nosotros, las respuestas sobre si conocen casos de compraventa fraudulenta de contratos de trabajo son poco concluyentes.

3.5. Círculos viciosos: "no hay trabajo porque no hay papeles y no hay papeles porque no hay trabajo"

Por último, mencionamos un problema legal, repetidamente mencionado por distintos colectivos de apoyo a inmigrantes, que constituye un auténtico círculo vicioso. Con mucha frecuencia, los inmigrantes en situación irregular son rechazados por los empleadores, quienes no les ofrecen contratos debido a que se encuentran en situación irregular, conminándoles a que regresen si regularizan su situación. Por su parte, cuando acuden a los servicios de Extranjería, se les solicita que aporten una oferta de contrato de trabajo como requisito previo a la concesión del correspondiente permiso de residencia y trabajo. De esta manera, la regulación legal se convierte en un auténtico círculo vicioso de difícil salida.

El 100\% de los entrevistados manifiesta que se trata de un gravísimo problema para el colectivo inmigrante. Ninguna otra cuestión de las planteadas en las entrevistas ha generado tanto consenso como ésta.

Es el verdadero problema de la inmigración.

(Florence, mujer camerunesa de 44 años con formación universitaria).

¿Qué fue antes, el huevo o la gallina?

(Hassan, hombre marroquí de 45 años con estudios universitarios).

Si no tienes papeles ni te reciben en la mayoría de procesos de selección y tus posibilidades son casi nulas, porque el empleador pasa de hacer todos los trámites.

(Hasna, mujer marroquí de 27 años con título de Formación Profesional).

${ }^{44}$ Informe lceberg (2009: 236 y ss.). 


\section{Conclusiones}

En primer lugar, consideramos que el círculo vicioso de la inmigración constituye el mayor obstáculo en el proceso de integración laboral. Existen varias claves en este problema: la legislación sobre extranjería sitúa la obtención de un contrato de trabajo como la vía más accesible de regularización jurídica. Sin embargo, cuando los potenciales empleadores reciben a un inmigrante irregular, tienden a rechazar la posibilidad de emplearlo. Creemos que en algunos casos actúan de ese modo movidos por el desconocimiento sobre la normativa vigente. Pero, además, existen otras posibles causas de esta actitud; si accedieran a ofrecer un contrato a un inmigrante en situación irregular, dicho contrato quedaría condicionado a la efectiva obtención de los correspondientes permisos de residencia y trabajo. Para obtenerlos, el inmigrante deberá solventar con éxito varios trámites de gran complejidad, tales como la obtención del denominado Informe de Arraigo, que en ocasiones demora varios meses. La consecuencia es un proceso largo y de resultado incierto. Seguramente, salvo en casos muy concretos, no existen muchos empleadores dispuestos a asumir dicho proceso. La consecuencia de esto es que un alto número de inmigrantes son rechazados por las empresas una y otra vez por carecer de documentación, y la desesperación va creciendo en muchos de ellos, que se estancan en sectores informales, como la venta ambulante. Creemos que constituye un auténtico deber ético de las instituciones $y$, en general, de las sociedades de acogida, dar respuesta a este problema.

Así mismo, opinamos que la precarización de las condiciones de trabajo, que no sólo afecta a inmigrantes, sino también a muchos miles de personas autóctonas, debe ser traída a un lugar preferente en el debate social. Reforzar la Inspección de Trabajo y llevar a cabo una apuesta más decidida en esta línea por parte del Ministerio Fiscal podría intensificar la lucha contra la precarización de las condiciones de trabajo, muy especialmente en el ámbito de las mujeres inmigrantes irregulares empleadas de hogar en régimen interno. La situación de muchas de ellas podría ser calificada sin exageración como la esclavitud del siglo XXI.

Otro de los principales problemas evidenciados apunta a la necesidad de luchar contra las actitudes discriminatorias hacia los inmigrantes. Los centros educativos, imbuidos en un creciente proceso de multiculturalidad, ofrecen un marco privilegiado para educar en actitudes de respeto, tolerancia y valoración de la diversidad.

En lo relativo a la normativa y al papel de las AA.PP., consideramos que se trata de un ámbito legislativo complejo que regula una realidad altamente cambiante. No obstante, creemos que las administraciones deberían hacer un esfuerzo por 
sistematizar, clarificar y difundir la normativa vigente en materia de extranjería, a fin de que los inmigrantes conozcan mejor sus derechos y obligaciones. Respecto a los recursos, ayudas y servicios de orientación para el autoempleo, una gran mayoría de los inmigrantes declara no conocerlos. Así pues, también en este aspecto consideramos que las AA.PP. deberían hacer un mayor esfuerzo divulgativo, dadas las posibilidades que ofrece el autoempleo para generar riqueza en nuestro país y ofrecer soluciones a un colectivo con altas cifras de desempleo y una urgente necesidad de regularización jurídica.

Por último, creemos que tanto el colectivo inmigrante como ONGs y AA.PP. deben continuar redoblando esfuerzos por llevar a cabo un proceso de mayor y mejor cualificación que posibilite un más fácil y exitoso camino de integración profesional.

\section{Bibliografía}

ANEAS, A. y DONOso, T. (2008), "Estudio sobre los procesos de integración laboral de los inmigrantes", Revista española de orientación y psicopedagogía, Vol. 19, $\mathrm{N}^{\circ} 1,1^{\text {er }}$ Cuatrimestre, pp. 51-60.

Ariza, J.A., Morales, A.C. y Morales, E. (2010), "Condiciones laborales de los inmigrantes en la Unión Europea: un estudio comparado", Revista de Fomento Social 65, 205-233.

BaldWIN-EdWARDS, M. (2002), "Immigration and The Welfare State: A European Challenge to American Mythology", Simposio Internacional: Europa y el Mediterráneo. Políticas de Inmigración, Barcelona, Universidad Pompeu Fabra e Instituto Catalá de la Mediterránea.

BazzACO, E. (2008), "La Inmigración en España: Racismo Institucional y Racismo Social", Papeles Panorama n ${ }^{\circ}$ 103, pp. 75-84.

BonilLA, M. (2003), Programa Andino de DD.HH. en Revista de Aportes Andinos $\mathrm{n}^{\circ} 7$, octubre.

Bustamante, J. (2003), "La Construcción Social de la Vulnerabilidad de los Inmigrantes", en GODENAU, D. y ZaPATA V. M. (coord.), La Inmigración Irregular, Aproximación Multidisciplinar, Ed. Cabildo Insular de Tenerife. 
GodenAU, D., (2003), "La Inmigración Irregular Interpretada desde una Visión Económica", en GODEnAU, D. y ZaPATA V. M. (coord.), La Inmigración Irregular, Aproximación Multidisciplinar, Ed. Cabildo Insular de Tenerife.

GozAlves (1995), "La Inmigración Irregular de Africanos en España, Balances y Perspectivas". Coloquio internacional Migration clandestine: enjeux et perspectives, Association Marocaine d'Etudes et de Recherches Sur les Migrations. Rabat.

IZqUieRDO, A. (2003), Inmigración: Mercado de Trabajo y Protección Social en España, CES, Madrid.

LALONDE, R. y TOPEL, R. (1995), "Economic Impact of International Migration and the Economic Performance of Migrants". Handbook of Population and Family Economics. Elsevier Science.

LóPEZ, A. M. (2003), "La Inmigración Irregular en la Investigación Sociológica", en GOdenau D. y ZapATA V. M. (coord.), La Inmigración Irregular, Aproximación Multidisciplinar, Ed. Cabildo Insular de Tenerife.

- (2005), "El Control de la Inmigración: Política Fronteriza, Selección del Acceso e Inmigración Irregular". Arbor, Ciencia, Pensamiento y Cultura 713.

Martínez Martínez, J. (2006), "Pensar la integración de los inmigrantes: la sociedad española al comienzo del siglo XXI", Revista de Fomento Social 61, 361-392.

MASSEY, D.S. (1993), "Theories of International Migration: a Review and Appraisal", Population and Development Review, vol. 19, pp. 431-466.

MORENO, F. y BRUQUetAS, M., 2011, Inmigración y Estado de Bienestar en España. Colección de Estudios Sociales n 31 . Ed. Obra Social "La Caixa".

Noll, G. (2010), "Why Human Rights Fail to Protect Undocumented Migrants?", European Journal of Migration and Law $\mathrm{n}^{\circ}$ 12, pp. 241-272.

Plataforma Andalucía Acoge (2009), Informe lceberg. Estudio del Proceso y Evolución de la Vida Laboral de Personas Extranjeras no Comunitarias en Andalucía.

Solanes, A., (2006), "Integración sin Derechos, de la Irregularidad a la Participación", Cuadernos Electrónicos de Filosofía del Derecho, n 14. 
SolÉ, C. et al. (2000), "El Impacto de la Inmigración en la Sociedad Receptora", Reis 90/00, pp. 131-157.

TJeRNBERG, M. (2010), "The Economy of Undocumented Migration: Taxation and Access to Welfare". European Journal of Migration and Law (12), pp. 149-171.

Villagómez, E. y Otero E. (2008), "Las experiencias de éxito de promoción de la integración laboral de los inmigrantes y de colectivos hasta ahora excluidos total o parcialmente", Revista del Instituto de Estudios Económicos, nos 2 y 3, pp. 167-190. 